\title{
A NOTE REGARDING ABSTRACT OPERATORS AND PASSIVE NETWORKS*
}

\author{
By E. BELTRAMI (State University of New York at Stony Brook)
}

The purpose of this note is to point out two facts regarding the connection between scattering and impedance formalisms for passive electrical networks. Both facts follow directly from the theory of monotone operators and apparently have not been noted before.

A passive network is defined here to be a linear monotone map $L$ on a dense subset of $L_{2}$ :

$$
\operatorname{Re}(v, L v) \geq 0
$$

for all inputs $v$ in the domain of $L$ (voltages). Usually the domain is taken to be the Schwartz space $D$ of $C^{\infty}$ functions. More generally, one deals with $H_{0}$-valued $L_{2}$ functions, where $H_{0}$ is a Hilbert space, and condition (1) is generalized to read

$$
\operatorname{Re} \int_{-\infty}^{t}(v, L v)_{0} d t \geq 0
$$

for all $t>-\infty$. When $H_{0}$ is $R^{n}$ then $L$ is said to describe an $n$-port network; otherwise, following Zemanian [1], the system is termed a Hilbert port. In this paper we restrict ourselves to scalar functions only, and to the case $t=+\infty$. This is done simply for expediency of notation and the results below remain valid in the general case. Notice also that we make no use of the notions of causality, translation invariance and continuity.

It is implicit in the above definitions that $L$ is single-valued. This condition already excludes certain standard networks, such as the two-port ideal transformer whose inputs

$$
v=\left(\begin{array}{l}
v_{1} \\
v_{2}
\end{array}\right)
$$

transform into the currents

$$
i=\left(\begin{array}{l}
i_{1} \\
i_{2}
\end{array}\right)
$$

while satisfying the relations $v_{2}=v_{1}, i_{2}=-i_{1}$. In an effort to extend the notion of passive operator to handle such cases, Youla, Castriota and Carlin [2] introduced the concept of an augmented network which is obtained by cascading a simple one-ohm resistor with the network defined by $L$ (see Fig. 1). Specifically, the input $v$ is replaced by the "incident voltage" $a=v+L v$. This allows us to define a single-valued mapping $S$ by $S a=v-L v$, which is the "reflected voltage". In fact, the correspondence $L \rightarrow S$

* Received August 20, 1971. 


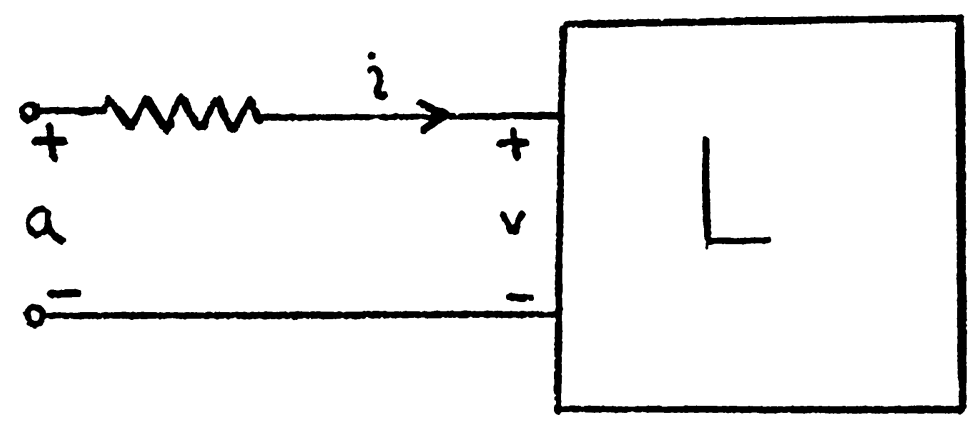

Fig. 1.

is obtained by a Cayley transform and $S$ is easily shown to be a contraction. In this new formalism, the network is now said to be scatter-passive.

However, in order to make the definition of $S$ useful one would like its domain also to be dense in $L_{2}$. That is, the problem is to insure that the equation

$$
a=v+L v
$$

is uniquely solvable in the domain of $L$ for all $a$ in a dense subset of $L_{2}$. This property is explicitly assumed as an axiom in [2]. In other treatments of network theory, the question of existence is made to depend on the additional hypothesis of translation invariance and continuity (in the Schwartz topology of $D$ ) for the operator $L$ (e.g., [3]).

One of our objectives here is to point out that if the single-valued $L$ is simply maximal monotone (i.e., does not possess a non-trivial extension to a monotone operator on a larger space), then indeed (3) possesses a unique solution. This fact is due to Phillips [4] and is a basic result in the theory of dissipative operators. Note that in general $L$ need not be bounded.

The second point that interests us here is the possibility of going back. That is, if we are given a linear contraction $S$-a scatter-passive system-then when is it true that one can define an equivalent $L$-passive formulation for the same network? The ideal transformer example shows that in general this cannot be done. It is known, however, that if $I+S$ is invertible on its range, then a single-valued and linear monotone map $L$ can be defined with a domain equal to the range of $I+S$. In fact, if $v=(a+S a) / 2$, then we let $L v=(a-S a) / 2$. What has not been observed before (at least in network circles) is that a sufficient condition for the invertibility of $S$ is that the range of $I+S$ be dense in $L_{2}$. This fact, which is also due to Phillips [4], is interesting for several reasons. First of all, it guarantees that the domain of $L$ will itself be dense. Second, if the domain of $S$ fills out $L_{2}$ then $L$ will actually be a maximal monotone operator.

\section{REFERENCES}

[1] A. H. Zemanian, The Hilbert port, SIAM J. Appl. Math. 18, 98-138 (1970)

[2] D. C. Youla, L. J. Castriota and H. J. Carlin, Bounded real scattering matrices and the foundations of linear passive network theory, IEEE PGCT-6, 102-124 (1959)

[3] M. R. Wohlers and E. J. Beltrami, Distribution theory as the basis of generalized passive-network analysis, IEEE PGCT-12, 164-169 (1965)

[4] R. S. Phillips, Dissipative operators and hyperbolic systems of partial differential equations, Trans. AMS 90, 193-254 (1959) 